\title{
Complexes Comprised of Charged Dendrimers, Linear Polyelectrolytes, and Counterions: Insight through Coarse-Grained Molecular Dynamics Simulations
}

\author{
Sergey V. Lyulin, ${ }^{\dagger}$ Ilpo Vattulainen, ${ }^{\ddagger, \S, \|}$ and Andrey A. Gurtovenko*, ${ }^{*}$ \\ Institute of Macromolecular Compounds, Russian Academy of Sciences, Bolshoi Prospect 31, \\ V.O., St. Petersburg 199004, Russia; Department of Physics, Tampere University of Technology, \\ P.O. Box 692, FI-33101 Tampere, Finland; Helsinki University of Technology, \\ P.O. Box 1100, FI-02015 HUT, Finland; MEMPHYS-Center for Biomembrane Physics, University of \\ Southern Denmark, Odense, Denmark; and Computational Biophysics Laboratory, Institute of \\ Pharmaceutical Innovation, University of Bradford, Bradford, West Yorkshire, U.K.
}

Received April 2, 2008; Revised Manuscript Received April 30, 2008

\begin{abstract}
Given the exceptional potential of dendrimer macromolecules for numerous biomedical applications, we performed extensive coarse-grained molecular dynamics simulations to investigate the role of electrostatic interactions in complexes comprised of cationic dendrimers with oppositely charged linear polyelectrolytes. For this purpose, we varied the nature of polyelectrolytes by considering both mono- and divalent chains and studied these cases for different valency of counterions. The dielectric properties of the solvent were also varied systematically. The counterions as well as solvent molecules were explicitly included in the model. It turned out that the complexation of a linear polyelectrolyte with a dendrimer leads to a remarkable condensation of the complex. Furthermore, formation of the complex gives rise to a considerable dehydration of the chain, the dehydration becoming more pronounced when the electrostatic interactions strengthen. Thus, charged dendrimers clearly demonstrate ability for efficient compaction of guest chains and protective screening of the chains from the surrounding medium, the two well-known prerequisites for vehicle-mediated delivery of drugs and genes into cells. In addition, our study indicates noticeable effects of counterions on the structure of dendrimer-chain complexes. These effects become more pronounced with increasing strength of electrostatic interactions.
\end{abstract}

\section{Introduction}

The delivery of a drug to the site of its action is a major challenge for virtually all drug candidates. In particular, the efficient and safe delivery of therapeutic nucleic acids has remained as one of the main problems in gene therapy. A way forward is to resort to the use of nanosized delivery vehicles which would effectively compact and protect a guest drug molecule, facilitating its trafficking to the target.

Dendrimers with their unique molecular architecture and related properties seem to be ideal candidates for these purposes. ${ }^{1}$ The symmetrical branching structure of dendrimers ${ }^{2}$ is a key for direct control over dendrimer's size, shape, interior density, and surface functionality, allowing dendrimers to serve both as nanocontainers and as surface-active colloid particles. Consequently, they have been extensively employed for biomedical applications. For example, polyamidoamine (PAMAM) dendrimers protonated in aqueous solution under physiological conditions $^{3}$ are routinely used as synthetic delivery vectors for gene transfection. ${ }^{4-7}$ Furthermore, they have been used for delivery of a variety of small drug molecules such as the anticancer drug cisplatin. ${ }^{1,8}$

From a physicochemical point of view, the idea behind the applicability of dendrimers for gene delivery is rather simple. As a nucleic acid strand carries a large negative charge in the backbone, its effective compaction can be based on strong electrostatic interactions with a positively charged synthetic vector such as e.g. cationic lipids, cationic polymers, and

\footnotetext{
* Corresponding author. E-mail: A.Gurtovenko@bradford.ac.uk.

† Institute of Macromolecular Compounds.

$\div$ Tampere University of Technology.

$\S$ Helsinki University of Technology.

"University of Southern Denmark.

${ }^{\perp}$ University of Bradford.
}

PAMAM dendrimers in aqueous solution. ${ }^{9}$ Therefore, the high positive surface charge of protonated dendrimers, combined with dendrimer's low toxicity (especially for low-generation dendrimers $^{10-12}$ ) renders them suitable for gene transfection. As far as drug molecules are concerned, they can be retained in the dendrimer's interior either through electrostatic interactions or through hydrogen bonding with protonated amide groups. ${ }^{1}$

Given dendrimers' exceptional potential for numerous biomedical and technological applications, it is not surprising that dendrimer macromolecules have attracted considerable attention of theoreticians over the past two decades. First computational models of dendritic polyelectrolytes ${ }^{13-17}$ relied on the simplified, implicit description of counterions within the linearized Poisson-Boltzmann (Debye-Hückel) theory and on the treatment of solvent as continuum medium. Despite their obvious limitations, these studies provided first insight into the properties of charged dendrimers.

Only very recently have much more involved computational models emerged. The development in the field has been twofold. Starting from detailed approaches, increasing computer power and highly efficient simulation codes have allowed one to model the behavior of dendrimer molecules in aqueous solution at full atomic resolution. ${ }^{18-21}$ Such simulations have usually aimed to establish a direct link with experimental data, providing unprecedented details into the microscopic structure, hydration, and electrostatic properties of dendrimers. However, because of their accurate nature, atomistic simulation models are also computationally intensive, meaning that the time and length scales feasible for atomistic approaches are very limited. This is a major problem especially in charged systems where equilibration and residence time scales of counterions and salt ions can be exceedingly large. For this reason, a commonly used strategy is to consider more generic features of dendritic polyelectrolytes through (coarse-grained) models of lower 
resolution. These models go beyond the Debye-Hückel approximation, allowing one to describe ions explicitly ${ }^{2-28}$ even if the solvent is of implicit nature. Furthermore, recent work ${ }^{23,24}$ has shown how one can also account for the discrete nature of solvent through explicit nonpolar ${ }^{23}$ and polar ${ }^{24}$ solvent molecules. These studies demonstrate that the level of coarse-grained modeling has reached a state where all relevant dendrimer properties can be modeled in a realistic fashion as long as one is interested in qualitative rather than quantitative features of the system. What is more, as coarse-grained models are computationally considerably less demanding than atomistic descriptions, they can provide major added value in studies of generic properties of dendrimers. This idea has been employed very recently in cases where the aim has been to systematically consider effects due to the size and charge density of dendrimers, ${ }^{25,26}$ the strength of electrostatic interactions, ${ }^{22,23}$ and the concentration of salt and valency of salt ions ${ }^{27}$ and counterions. ${ }^{28}$

While there has been significant progress in molecular modeling of solvated charged dendrimers, considerably less attention has been paid to simulations of complexes between dendrimers and linear polyelectrolytes. ${ }^{29-33}$ Nonetheless, such studies are crucial to understand the complexation of cationic dendrimers with nucleic acids and drug molecules, since the complexation represents the very first step in vehicle-mediated gene and drug delivery. Furthermore, the complexation of polyelectrolytes with macroions is also of considerable fundamental interest due to the profound prospects of understanding how charged macromolecules interact with charged polyelectrolytes.

The above view can be elaborated in more detail by noting that most computational studies of charged dendrimers to date have employed very simplified models in which counterions of both dendritic and linear polyelectrolytes have been treated implicitly, and the solvent has been replaced with a structureless medium described by some dielectric permittivity. ${ }^{29,30,32,33}$ This approach may be problematic when applied to dense structures such as dendrimers, where the Debye-Hückel approximation can easily break down as charges approach each other and their distance goes below the Bjerrum length. Indeed, recent computational studies of single charged dendrimers ${ }^{22,23}$ with explicit counterions have clearly demonstrated that counterion condensation upon increasing strength of electrostatic interactions is able to cause noticeable changes in dendrimer's structure, which cannot be accounted for within the Debye-Hückel approximation.

The limited understanding of the structures of charged dendrimers is further underlined by the major computational challenge associated with state of the art atomistic studies. In a very recent work, Maiti and Bagchi $^{31}$ studied DNA-PAMAM complexes through atomistic simulations including counterions and water molecules in an explicit fashion. While this landmark paper presents one of the most significant efforts in the field, its very high computational load (a single system under study consisted of about 75000 atoms and was simulated for $20 \mathrm{~ns}$ with a time step of $2 \mathrm{fs})^{31}$ prohibits systematic studies of various factors that would affect the structures of the complexes, e.g., ionic strength and solvent's dielectric properties. Generic, coarse-grained models with explicit solvent and counterions are best suited for such large-scale simulations. However, to our knowledge, models of this kindhavenotbeenemployed formolecularsimulations of dendrimer-linear polyelectrolyte complexes.

In this study, we present extensive molecular dynamics simulations of complexes comprised of a generic (coarse-grained) charged model for a dendrimer of the fourth generation and a polyelectrolyte chain of opposite charge. Counterions of both the dendrimer and the linear polyelectrolyte as well as solvent molecules are explicitly incorporated in the model. We consider the case of relatively short chains, meaning that the overall charge of a chain is several times smaller than the total charge of a dendrimer. The strength of electrostatic interactions is systematically varied and its influence on the properties of dendrimer-chain complexes is monitored in detail. Special attention is paid to understand the effects associated with counterions; the issues related to the valency of charges of counterions and polyelectrolyte chains are also addressed. Overall, our study demonstrates that charged dendrimers have an ability for efficient compaction of guest polyelectrolyte chains and for protective screening of the chains from the surrounding medium.

\section{Model and Simulation Setup}

We have performed molecular dynamics (MD) simulations of dendrimers of generation four in their protonated state, so that all beads of the terminal (fourth) generation were positively charged with unit charge. We employed the same freely jointed "bead-and-spring" model without angle and torsion potentials as in ref 23; the model trifunctional dendrimer does not have spacers between branching points and consists of 94 beads, of which 48 were terminal positively charged beads. Such a charge pattern corresponds to physiological (neutral) $\mathrm{pH}$ conditions where the primary amines of a PAMAM dendrimer get protonated. ${ }^{3}$

A linear polyelectrolyte chain was placed nearby the dendrimer and was modeled at the same footing as a dendrimer with the use of the freely jointed model. The chain in the model was chosen to have 10 negatively charged beads with charge $-1 e$ or $-2 e$. This level of description allows us to study the coupling between the valency of the chain and the valency of the counterions. Yet even in the case of the divalent chain the overall charge of a dendrimer exceeds the charge of a polyelectrolyte chain, implying that we consider here the limit of rather short chains. In practical terms, such short linear monovalent polyelectrolytes can be considered as a generic model for small drug molecules as well as short single-stranded DNA fragments (see discussion at the end of the section).

The dendrimer-chain complex was solvated in a box of $\sim 9300$ nonpolar solvent molecules (beads). An appropriate number of counterions of dendritic and linear polyelectrolytes were added to the system by assigning charges to some randomly chosen solvent particles. As divalent ions such as calcium and magnesium are highly biologically relevant in cellular environments, both monovalent and divalent counterions were considered. A polymer volume fraction in the system was set to about $1 \%$.

The bonds between dendrimer branching points and between chain beads were modeled as harmonic springs with the potential energy $U=1 /{ }_{2} k\left(l-l_{0}\right)^{2}$, where $l_{0}$ is the bond length. The spring constant $k=10^{3} k_{\mathrm{B}} T / l_{0}{ }^{2}$ was taken from previous studies. ${ }^{34,23}$ Shortrange nonbonded interactions were described by the standard Lennard-Jones potential $U_{\mathrm{LJ}}=4 \epsilon\left[(\sigma / r)^{12}-(\sigma / r)^{6}\right]$ with $\epsilon=$ $0.3 k_{\mathrm{B}} T$. The bead-bead interaction diameter $\sigma$ was set to $0.8 l_{0}$; the diameter was taken to be the same for dendrimer beads, chain beads, counterions, and solvent molecules. The LennardJones potential was cut off at $r_{\mathrm{c}}=2^{1 / 6} \sigma$ and shifted to zero, ${ }^{35}$ describing the system under good solvent conditions. The longrange electrostatic interactions between the charged beads (terminal dendrimer beads, chain beads, and counterions) were handled using the particle-mesh Ewald (PME) method. ${ }^{36,37}$ The real-space cutoff was $4.55 l_{0}$, and the Fourier grid spacing was set to $0.33 l_{0}$. The splines of the sixth order were used for interpolation. Following refs 38 and 39, the density was set to $1.688 l_{0}^{-3}$ (or to $0.864 \sigma^{-3}$ ). The linear size of a cubic simulation box was set to $17.75 l_{0}$, and periodic boundary conditions were applied in all three dimensions.

In all, we studied three different types of dendrimer-chain complexes. (i) A charged dendrimer, a monovalent chain, and monovalent counterions; the system is referred to as DCI-111 system. Here and below the three letters DCI stand for dendrimer-chain-ions and are followed by three figures which denote the valency of charges for the three principal components of the system. In this manner, DCI-111, for example, means 
Table 1. Summary of Simulated Systems

\begin{tabular}{lccccccc}
\hline system $^{a}$ & $Q_{\text {total }}^{\text {dend }}$ & $N_{\text {1on }}^{\text {dend }}$ & $Q_{\text {1on }}^{\text {dend }}$ & $Q_{\text {total }}^{\text {chain }}$ & $N_{\text {1on }}^{\text {chain }}$ & $Q_{\text {10n }}^{\text {chain }}$ & $N_{\text {total }}^{\text {sol }}$ \\
\hline DCI-111 & $+48 e$ & 48 & $-e$ & $-10 e$ & 10 & $+e$ & 9265 \\
DCI-121 & $+48 e$ & 48 & $-e$ & $-20 e$ & 20 & $+e$ & 9255 \\
DCI-112 & $+48 e$ & 24 & $-2 e$ & $-10 e$ & 5 & $+2 e$ & 9294 \\
dendrimer & $+48 e$ & 48 & $-e$ & & & & 9285 \\
1V chain & & & & $-10 e$ & 10 & $+e$ & 980 \\
2V chain & & & & $-20 e$ & 20 & $+e$ & 970
\end{tabular}

${ }^{a}$ Here $Q_{\text {total }}^{\text {dend }}$ and $Q_{\mathrm{total}}^{\text {chain }}$ are the total charges of a dendrimer and a polyelectrolyte chain, respectively; $N_{\mathrm{ion}}^{\mathrm{dend}}$ and $N_{\mathrm{ion}}^{\text {chain }}$ are the total number of dendrimer's and chain's counterions; $Q_{\text {1on }}^{\text {dend }}$ and $Q_{10 n}^{\text {chain }}$ are the charges of these counterions; and $N_{\text {total }}^{\mathrm{sol}}$ is the total number of solvent molecules in a system.

that peripheral dendrimer beads, chain beads, and counterions are all monovalent. (ii) A charged dendrimer, a divalent chain, and monovalent counterions (the system DCI-121). (iii) A charged dendrimer, a monovalent chain, and divalent counterions of both the dendrimer and the chain (the system DCI-112).

All simulated systems are summarized in Table 1. For reference, we also carried out simulations of single charged dendrimers without a chain (system dendrimer in Table 1) and single linear polyelectrolytes in solution without a dendrimer (both monovalent and divalent chains; see systems $1 \mathrm{~V}$ chain and $2 \mathrm{~V}$ chain in Table 1).

The simulations for all the studied systems were repeated six times with different values of the Bjerrum length $\lambda_{\mathrm{B}}=e^{2} /$ $4 \pi \varepsilon_{0} \varepsilon_{\mathrm{s}} k_{\mathrm{B}} T$, which is a measure of the strength of electrostatic interactions in the system. In all, the value of $\lambda_{\mathrm{B}} / l_{0}$ was varied as follows: $0.2,0.6,0.8,1.0,3.2$, and 8.0. The variation in the Bjerrum length $\lambda_{\mathrm{B}}$ can be interpreted as a change in the dielectric permittivity of the solvent $\varepsilon_{\mathrm{S}}$ since $\lambda_{\mathrm{B}} \sim 1 / \varepsilon_{\mathrm{S}}$. Small values of $\lambda_{\mathrm{B}}$ reflect liquids with large $\varepsilon_{\mathrm{s}}$ (for water $\varepsilon_{\mathrm{s}} \approx 80$ ), while large values of $\lambda_{\mathrm{B}}$ correspond to nonpolar liquids such as the hydrophobic core inside cell membranes, where $\varepsilon_{\mathrm{s}} \approx 2-5$ is comparable to conditions in vacuum.

All dendrimer-chain complexes with different Bjerrum lengths were first equilibrated for $10^{6}$ time steps in the $N V T$ ensemble with the time step $\Delta t=2.4 \times 10^{-3} \tau_{0}$, where $\tau_{0}=$ $l_{0}(m / \epsilon)^{1 / 2}$ is the time unit of the model ( $m$ is the mass of a bead). The temperature was set to $\epsilon /\left[0.3 k_{\mathrm{B}}\right]$ and was controlled with the use of the Berendsen thermostat ${ }^{40}$ with a coupling time constant of $1.2 \times 10^{-1} \tau_{0}$. All bond lengths during NVT runs were constrained using the SHAKE algorithm. ${ }^{41}$ At this point it was noticed that the use of the thermostat and bond length constraints induced some (although rather subtle) artifacts seen in particular in translational properties of polymers. Therefore, to get rid of these artifacts, after initial equilibration the thermostat and bond length constraints were switched off, and the time step was reduced by a factor of 2 . Data were then collected from production runs of $5 \times 10^{6}$ time steps in the NVE ensemble. All the simulations were performed using the Gromacs suite. ${ }^{42}$ In total, the simulations took more than $42000 \mathrm{~h}$ of CPU time.

The scales of the model are defined by $l_{0}$ (length), $k_{\mathrm{B}} T$ (energy), and $m$ (mass). The most relevant one in the present work is the length scale described by the Bjerrum length $\lambda_{\mathrm{B}}$, which we present as a dimensionless number through $\lambda_{\mathrm{B}} / l_{0}$. To get some flavor of how $\lambda_{\mathrm{B}} / l_{0}$ is related to realistic systems, the average size of the coarse-grained model dendrimer used in this study was compared to that of the corresponding dendrimer in full-atom representation. ${ }^{23}$ Using this recipe, one has $l_{0} \simeq 6.7 \AA$, so that the Bjerrum length $\lambda_{\mathrm{B}}$ for water amounts to $\sim 1.3 l_{0}$.

It is instructive to compare the persistence length $P$ of model polyelectrolyte chains employed in this study and that observed for DNA. For a monovalent chain at $\lambda_{\mathrm{B}}=l_{0}$ we found we $^{35,43}$ $P \simeq 1.37 l_{0} \simeq 9.18 \AA$. Experimental values ${ }^{44,45}$ for the persistence length of single-stranded DNA can scatter from 7.5
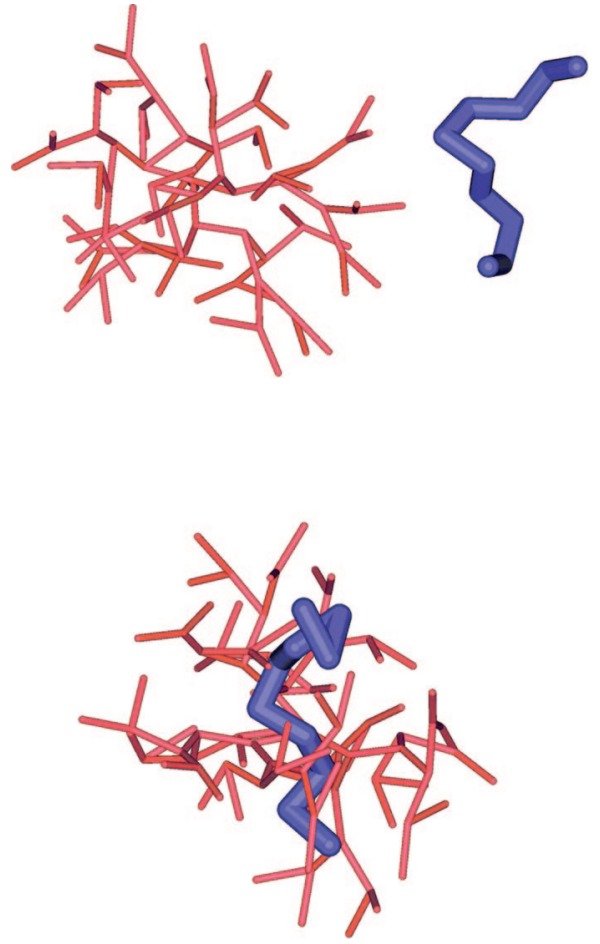

Figure 1. Representative configurations of the dendrimer-chain system (top) before and (bottom) after complexation.

to $30 \AA$; it is also sensitive to ionic strength of solution. ${ }^{45}$ Therefore, the stiffness of the model polyelectrolyte chain used turns out to be comparable with that of ssDNA. As far as a double-stranded DNA is concerned, its persistence length is considerably larger $(P \simeq 500 \AA),{ }^{46}$ so that our flexible chain model can hardly serve as an adequate description of a ds-DNA fragment.

\section{Results and Discussion}

A. Dendrimer and Chain Compaction upon Complexation. For all considered systems the electrostatic interactions between a cationic dendrimer and an anionic linear chain were found to be strong enough to lead to formation of a stable complex during equilibration; this holds for all considered values of the Bjerrum length. Rough estimates show that e.g. for the system DCI-111 with the smallest value of the Bjerrum length $\left(\lambda_{\mathrm{B}}=0.2 l_{0}\right)$ the electrostatic interactions are of an order of several $k_{\mathrm{B}} T$, so that electrostatics dominates over thermal fluctuations. The fast complexation is at least in part due to the fact that we consider relatively short chains (10 beads), whose total charge is smaller than that of a dendrimer. As a result, a dendrimer is able to electrostatically attract a chain and to take it up almost completely. Figure 1 depicts this situation through two representative configurations of a dendrimer-chain system before and after complexation.

The equilibration of the dendrimer-chain complex was monitored through the time evolution of the mean-square radius of gyration $\left\langle R_{\mathrm{g}}{ }^{2}\right\rangle$ (which is a measure of the overall size) of the entire complex. Additionally, we considered the $\left\langle R_{\mathrm{g}}{ }^{2}\right\rangle$ of a dendrimer and a chain separately. For full equilibration, we also required that mobile charges (corresponding to the counterions of the dendrimer and the chain) evolved to a stable distribution at long times. ${ }^{23}$ It turned out that both the size of the complex and the counterion distribution were fully equilibrated during the initial equilibration in the NVT ensemble and remained stable during the entire production run.

In the absence of a linear polyelectrolyte, the size of a dendrimer is found to express a pronounced nonmonotonic dependence on the Bjerrum length $\lambda_{B}$ (see Figure 2), in full 


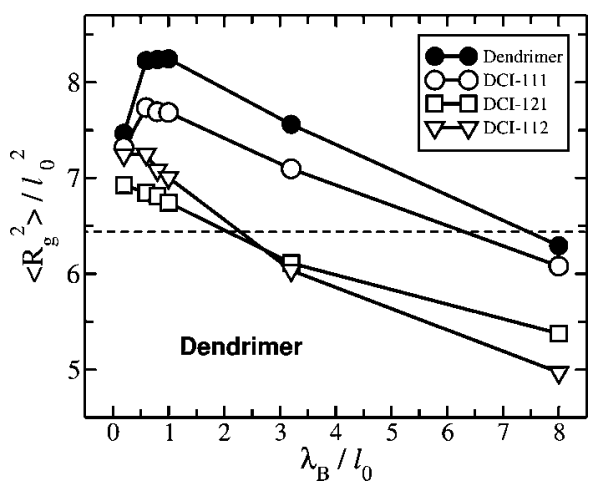

Figure 2. Average mean-square radius of gyration of dendrimers, $\left\langle R_{\mathrm{g}}{ }^{2}\right\rangle$, for the three considered types of complexes as a function of the Bjerrum length $\lambda_{\mathrm{B}}$. The $\left\langle R_{\mathrm{g}}{ }^{2}\right\rangle$ of a single (chain-free) dendrimer in solution is also presented; the dashed line shows the radius of gyration for an uncharged dendrimer. Here (and throughout the paper) error bars were estimated as the standard errors of mean by splitting trajectories into five pieces of $10^{6}$ time steps each. The error bars are not shown if they are of the same size as the symbols.

accordance with previous studies. ${ }^{23}$ The position of the peak in $\left\langle R_{\mathrm{g}}{ }^{2}\right\rangle$ is very close to the bond length, $\lambda_{\mathrm{B}} \simeq l_{0}$.

When the cationic dendrimer merges with the anionic chain, the complexation has a dramatic effect on the dendrimer's size, reducing it substantially. This is evident for all values of the Bjerrum length. Of particular interest is the case where $\lambda_{\mathrm{B}} / l_{0} \sim$ 1.0 , since in units of our model the Bjerrum length in water is about $1.3 l_{0}$ (see ref 23). For systems with such moderate values of $\lambda_{\mathrm{B}}$, the complexation of a dendrimer with a divalent polyelectrolyte chain leads to a striking reduction in the size of the complex (see Figure 2). Though a similar change in size is also found for the case with a monovalent chain, the effect is then less prominent. This effect is in line with intuitive expectations since at a fixed value of $\lambda_{\mathrm{B}}$ the electrostatic attraction of a dendrimer with a divalent chain is much stronger than with its monovalent counterpart.

A closer inspection reveals that complexation of a dendrimer with a monovalent polyelectrolyte decreases the dendrimer size by $\sim 8 \%$ at $\lambda_{\mathrm{B}} / l_{0} \sim 1.0$, while with a divalent chain the dendrimer size drops as much as $\sim 18 \%$. Divalent counterions considerably enhance the dendrimer's compaction upon complexation with monovalent chains; Figure 2 demonstrates this feature very clearly especially at large values of $\lambda_{\mathrm{B}} / l_{0}$ (cf. systems DCI-112 and DCI-121).

What is even more interesting is the fact that complexation changes the nature of dendrimer size dependence on the Bjerrum length. For the dendrimer-monovalent chain complex (DCI111), the mean-square radius of gyration still demonstrates nonmonotonic behavior. Such behavior, typical for single cationic dendrimers, has been shown to originate from the interplay between repulsive electrostatic interactions for the charged terminal dendrimer beads and counterion condensation onto a dendrimer. ${ }^{23}$ It seems that a monovalent chain adsorbed on a dendrimer does not change this picture. However, when divalent features join the game, the peak in $\left\langle R_{\mathrm{g}}{ }^{2}\right\rangle$ either decays substantially or disappears completely. This phenomenon is observed when the dendrimer forms a complex with a divalent chain with monovalent counterions and also when the chain is monovalent but the counterions are divalent (see Figure 2). This implies that the condensation of divalent charges (either free as in DCI-112 or connected into a chain as in DCI-121) is so strong that it screens the repulsion between the charged dendrimer beads. Thus, the size of a dendrimer can show both nonmonotonic and monotonic behavior for increasing strength of electrostatic interactions depending on the charge density of a polyelectrolyte chain and on the valency of counterions.
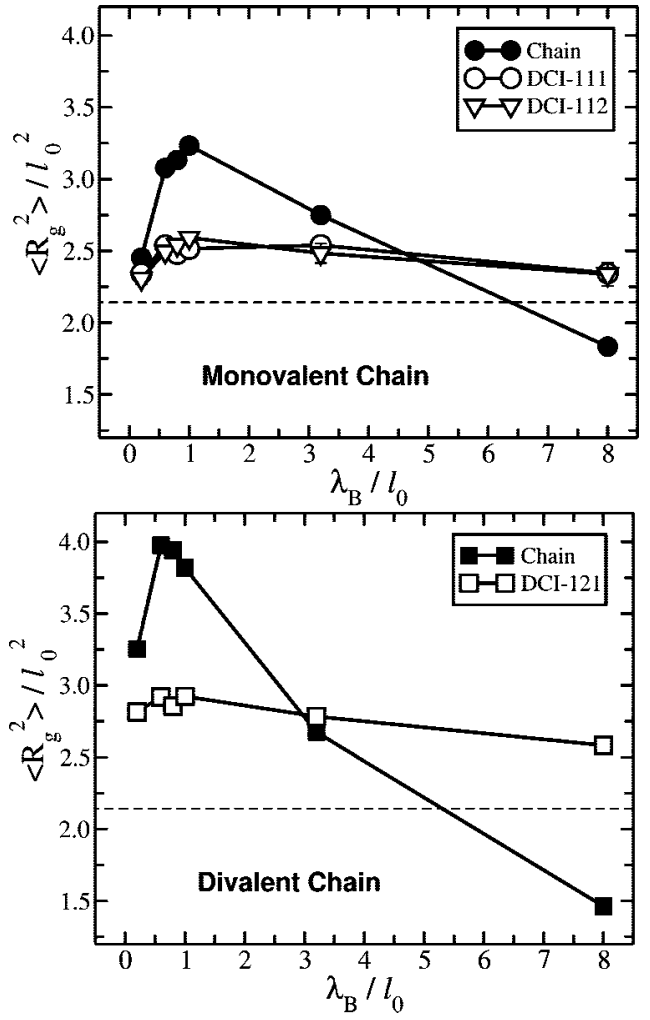

Figure 3. Average mean-square radius of gyration of linear polyelectrolytes, $\left\langle R_{\mathrm{g}}{ }^{2}\right\rangle$, in complexes and in solution as a function of the Bjerrum length $\lambda_{\mathrm{B}}$. Shown are results for (top) monovalent and (bottom) divalent chains. The dashed line shows the $\left\langle R_{\mathrm{g}}{ }^{2}\right\rangle$ for an uncharged chain.

Of great practical interest is the change in the size of a polyelectrolyte guest molecule embedded in the complex, since chain compaction is an important prerequisite for efficient drug and gene delivery. ${ }^{1,7}$ Figure 3 depicts the mean-square radii of gyration for (free) polyelectrolyte chains in the absence of a complex and for the same chains embedded in a complex. For free chains we find the well-known nonmonotonic dependence of $\left\langle R_{\mathrm{g}}{ }^{2}\right\rangle$ on the Bjerrum length, in full agreement with previous studies of linear polyelectrolytes with explicit counterions. ${ }^{35,47}$ The location of the peak for $\left\langle R_{\mathrm{g}}{ }^{2}\right\rangle$ was found to be at $\lambda_{\mathrm{B}} \sim 1.0 l_{0}$ for monovalent chains and at $\lambda_{\mathrm{B}} \sim 0.6 l_{0}$ for divalent chains. The origin of the nonmonotonic dependence seems to be similar to that for charged dendrimers: the increasing condensation of counterions with $\lambda_{\mathrm{B}}$. Interestingly, when the Bjerrum length becomes large, the radii of gyration of both charged dendrimers and linear polyelectrolytes get smaller than their uncharged counterparts (see Figures 2 and 3). It is most likely that ion condensation is responsible for such an effect as it can cause multipole attraction between different parts of a polymer (see also refs 22 and 23).

Complexation of linear polyelectrolytes with charged dendrimers leads to a considerable chain compaction. At $\lambda_{\mathrm{B}} \simeq l_{0}$ we find the size of polyelectrolyte chains in dendrimer-chain complexes to be about $20 \%$ smaller compared to a dendrimerfree solution (see Figure 3). Therefore, cationic dendrimers can be considered as rather effective agents for compaction of linear polyelectrolytes in water. However, it is interesting that this feature is evident only at small and moderate values of the Bjerrum length (up to $\lambda_{\mathrm{B}} \sim 4 l_{0}$ for monovalent chains and up to $\lambda_{\mathrm{B}} \sim 3 l_{0}$ for divalent chains) (see Figure 3). At large $\lambda_{\mathrm{B}}$ 's shrinking of a chain is achieved in a much more effective way by counterion condensation instead of its complexation with a dendrimer. The reason for that is twofold: The chain in a complex is not accessible for its counterions, and there are dendrimer-induced steric constraints preventing further compac- 


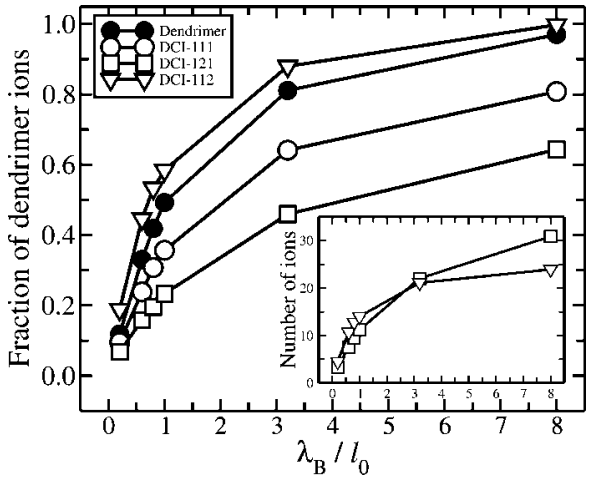

Figure 4. Average fraction of dendrimer counterions condensed onto a dendrimer in complex and in solution as a function of the Bjerrum length $\lambda_{\mathrm{B}}$. Inset shows the absolute number of condensed dendrimer counterions for the DCI-121 and DCI-112 systems.

tion of a chain after complexation. The important practical implication is that cationic dendrimers do not seem to be appropriate for effective chain compaction when the electrostatic interactions in a system are rather strong, e.g., when water is replaced by an organic solvent with much lower dielectric constant.

The character of $\lambda_{\mathrm{B}}$ dependence for the size of a chain becomes completely different in a dendrimer-chain complex: the mean-square radius of gyration of polyelectrolyte chains varies only slightly with $\lambda_{\mathrm{B}}$, especially for $\lambda_{\mathrm{B}} \geq 0.6 l_{0}$. Furthermore, $\left\langle R_{\mathrm{g}}{ }^{2}\right\rangle$ was found to be almost insensitive to ion valency. This is most likely again due to the fact that chain counterions cannot readily access the chain in a complex.

B. Counterion Condensation and Chain Dehydration. To clarify the role of counterion condensation, we calculated the fraction of adsorbed counterions for all the systems in question. For condensation we used the following simple criterion: a counterion was considered to be condensed on a dendrimer (chain) if it had a dendrimer (chain) bead in its first coordination (hydration) shell. Similar to previous studies, ${ }^{23,48-51}$ to evaluate the size of the hydration shell, we first calculated the radial distribution functions (RDFs) for beads of different types (dendrimer and chain beads, counterions, and solvent molecules). The radius of the first coordination shell was then extracted from the position of first minima in the RDFs. This radius was found to be the same for all beads in the system and equal to $\sim 1.3 l_{0}$.

In Figure 4 we present fractions of dendrimer counterions that have condensed on a dendrimer for the three dendrimer-chain systems studied, also including data for a single dendrimer (without a chain) in solution. First, one can see that for all systems fractions of condensed ions increase monotonously with the strength of electrostatic interactions. Second, for the complexes with monovalent counterions (DCI-111 and DCI121), ion condensation is considerably weaker compared to a single chain-free dendrimer; this effect is most pronounced for dendrimer complexes with divalent chains (see Figure 4). The observed decrease in condensation is due to the fact that complexation with an anionic chain partially compensates the charge of a cationic dendrimer. Such a compensation naturally depends on the valency of a chain: a divalent chain (DCI-121) neutralizes a dendrimer in a more efficient way than a monovalent chain (DCI-111) does; this explains the weaker ion condensation in the former case (see Figure 4). Meanwhile, the case with divalent counterions is completely different. Then the fraction of condensed divalent ions exceeds that for a single dendrimer. However, one should bear in mind that the number of divalent ions are twice smaller than the number of monovalent ions, so that the total number of ions condensed on a single
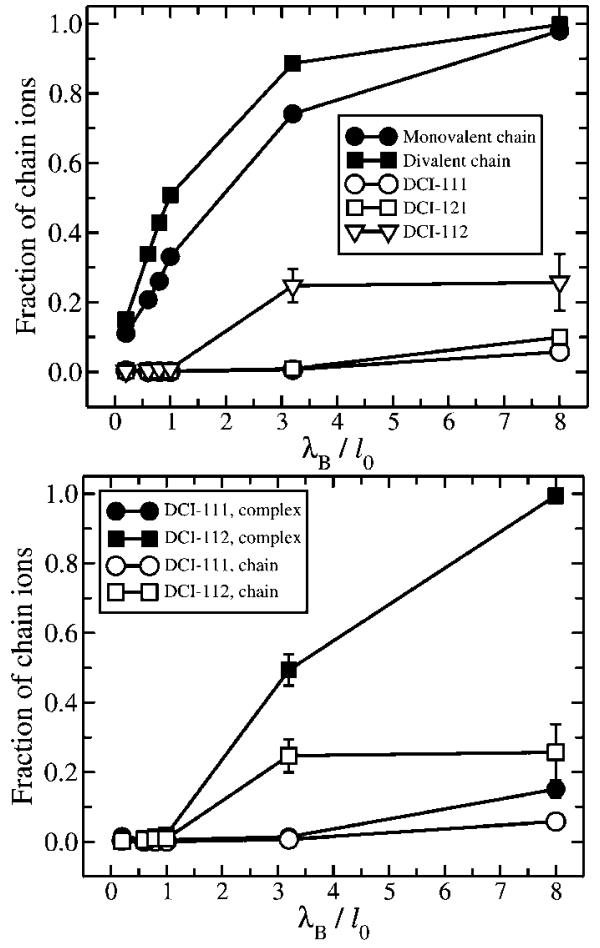

Figure 5. (top) Average fraction of chain counterions condensed on a polyelectrolyte chain in complex and in solution as a function of the Bjerrum length $\lambda_{\mathrm{B}}$. (bottom) Average fraction of chain counterions adsorbed on a chain and on a whole chain-dendrimer complex as a function of $\lambda_{\mathrm{B}}$.

chain-free dendrimer is still much higher than in the DCI-112 complex. Furthermore, Figure 4 shows the total numbers of condensed counterions for the DCI-121 and DCI-112 systems. Interestingly, they become equal at $\lambda_{\mathrm{B}} \simeq 3.0 l_{0}$, which coincides with the value of $\lambda_{\mathrm{B}}$ corresponding to the situation where these two systems have the same size (see Figure 2).

Figure 5 (top) illustrates condensation of chain counterions on a chain in complex and in solution. As expected, a dendrimer screens a polyelectrolyte chain from its counterions very efficiently, especially at small Bjerrum lengths $\left(\lambda_{\mathrm{B}} \leq l_{0}\right)$. Monovalent chain ions, in particular, are essentially not able to condense at all. This applies to both the chain and the dendrimer-chain complex (see Figure 5, bottom). However, this behavior is sensitive to ion valency: Divalent ions adsorb on a chain located inside a complex to a larger degree compared to monovalent ions. This difference between monovalent and divalent chain ions becomes much more pronounced when condensation on a dendrimer-chain complex as a whole is considered: One can observe even complete adsorption of the divalent chain counterions on a complex at $\lambda_{\mathrm{B}}=8.0 l_{0}$ (see Figure 5, bottom). Such behavior can also be related to the fact that for the system DCI-112 a very small tail of a polyelectrolyte chain protrudes from the complex (see also section $\mathrm{C}$ ).

The overall charge of a dendrimer-chain-ions complex has to be studied in more detail because of its major relevance for gene delivery: the positive charge of a complex as a whole is known to facilitate adsorption to the cell membrane. ${ }^{7}$ The charge of a dendrimer-chain system is also interesting in light of possible dendrimer overcharging when a charged dendrimer takes up more opposite charges than what is required for its complete neutralization.

To characterize the change in the total charge of a complex as the strength of electrostatic interactions increases, we calculated an effective charge of a complex in terms of $Q_{\text {complex }}$ $=Q_{\text {dend }}+Q_{\text {cond }}$, where $Q_{\text {dend }}$ is the total charge of charged dendrimer beads (for the considered dendrimer of fourth 

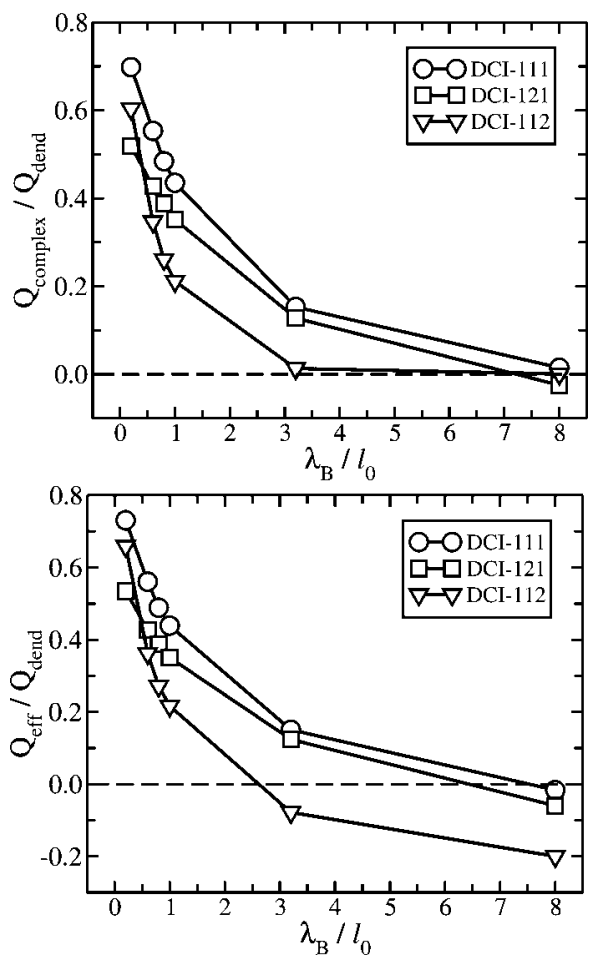

Figure 6. (top) Effective charge of a dendrimer-chain complex as a function of the Bjerrum length $\lambda_{\mathrm{B}}$. The charge $Q_{\text {complex }}$ is normalized by the total charge of dendrimer beads of the terminal G4 generation, $Q_{\text {dend. }}$. The dashed line corresponds to complete neutralization of the complex by counterions and a polyelectrolyte chain. (bottom) Effective charge of a cationic dendrimer $Q_{\text {eff }}$ considering that only oppositely charged anionic beads (dendrimer counterions and a polyelectrolyte chain) would condense on it; see text for details.

generation $Q_{\text {dend }}=+48$; see also Table 1) and $Q_{\text {cond }}$ is the total charge of beads condensed on a complex. Note that $Q_{\text {cond }}$ can arise from beads whose charges are opposite to a dendrimer (a polyelectrolyte chain and dendrimer counterions) but also from beads of the same charge (chain counterions; see Figure 5, bottom).

Figure 6 (top) shows $Q_{\text {complex }} / Q_{\text {dend }}$ as a function of the Bjerrum length $\lambda_{\mathrm{B}}$. In the case of monovalent counterions, a divalent chain (DCI-121) neutralizes the positive charge of a dendrimer more efficiently than a monovalent chain (DCI-111). Interestingly, one can also observe a slight inversion of the total charge of the DCI-121 complex at large $\lambda_{\mathrm{B}}=8.0 l_{0}$ (see Figure 6 , top). Furthermore, it turns out that divalent ions (the system DCI-112) are considerably more effective in neutralizing the charge of a complex as compared to their monovalent counterparts. This is evident especially at moderate and large values of the Bjerrum length. As a result, the positive charge of a complex completely vanishes already at $\lambda_{\mathrm{B}} \simeq 3.0 l_{0}$.

This finding has major implications in terms of drug and gene delivery. It signifies that an increase in the strength of electrostatic interactions leads to a major reduction in the positive charge of the dendrimer-chain complex and reduces its potential ability to bind to the plasma membrane. To better highlight the relevance of this view, let us stress that the dielectric permittivities of water and membrane hydrocarbon region are about 80 and $2-5$, respectively, and the dielectric permittivity thus decreases as one moves from the water phase through the membrane-water interface to the core of the membrane. Concurrently, the transition from water to membrane interior corresponds to a change from small to large $\lambda_{\mathrm{B}} / l_{0}$. Figure 6 (top) then depicts that the partitioning of the dendrimer-chain complex from water to membrane interior becomes more and more troublesome as the complex approaches the membrane.

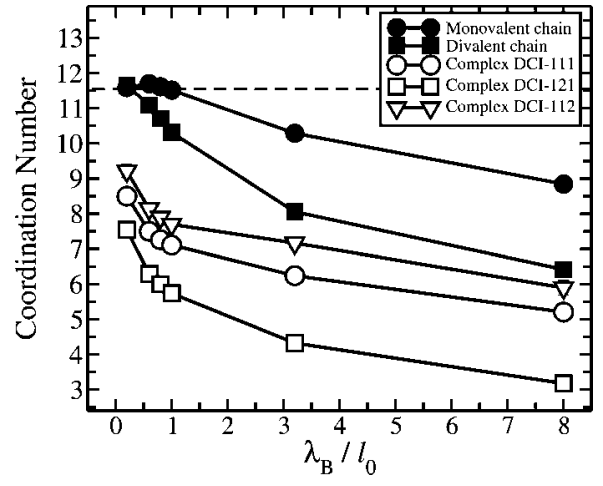

Figure 7. Average coordination numbers of polyelectrolyte chain's beads with solvent beads as a function of the Bjerrum length $\lambda_{\mathrm{B}}$. Shown are results for mono- and divalent chains in complex and in solution. The dashed line shows the hydration level of an uncharged polymer chain.

It is interesting to elucidate the role of chain counterions as they are of the same sign as dendrimer charges. In Figure 6 (bottom) we present the effective charge of a dendrimer, $Q_{\text {eff }}$, considering a situation where only oppositely charged beads (dendrimer counterions and a polyelectrolyte chain) would condense on it. A similar approach was employed in a recent study of complexation of a polyanion with a cationic micelle. ${ }^{52}$ While the curves for the systems with monovalent ions (DCI111 and DCI-121) remain almost unchanged compared to those in Figure 6 (top), one can witness a noticeable overcharging for a dendrimer in the presence of divalent ions at $\lambda_{\mathrm{B}} \geq 3.0 l_{0}$. This result contrasts with the findings of a recent simulation study by Majtyka and Klos, ${ }^{27}$ who studied single charged dendrimers under the presence of mono- and divalent salt and did not observe overcharging of dendrimers for a wide range of the Bjerrum lengths. The presence of charges connected to a chain may therefore be crucial for dendrimer's overcharging. Overall, the combined consideration of the top and bottom panels of Figure 6 clearly indicates that multivalent chain counterions are able to prevent a dendrimer-chain complex from overcharging when the electrostatic interactions are rather strong (see also Figure 5, bottom).

Complexation of a polyelectrolyte chain with a dendrimer should also affect the hydration level of a chain. To estimate this effect, we computed the average coordination numbers of chain beads with solvent molecules, i.e., the number of solvent beads within the first coordination shell around a chain bead. As seen in Figure 7, complexation with a cationic dendrimer leads to a remarkable dehydration of linear polyelectrolytes for all considered dendrimer-chain complexes. Interestingly, the dendrimer-induced dehydration for a divalent chain is larger than for its monovalent counterpart. For moderate values of the Bjerrum length, $\lambda_{\mathrm{B}} \sim l_{0}$, a monovalent chain in the DCI-111 complex loses $\sim 38 \%$ of solvent molecules from its first hydration shell ( $\sim 33 \%$ in the case of the DCI-112 complex). In turn, a divalent chain after complexation with a cationic dendrimer (DCI-121) gets dehydrated by $\sim 44 \%$ as compared to solution (see Figure 7). Taken together with the abovementioned screening of polyelectrolyte chains in a complex from their counterions (see Figure 5, top), the observed chain dehydration implies that a cationic dendrimer is indeed able to efficiently protect a guest molecule from the influence of the surrounding medium.

C. Localization of Chain in Complex. The degree of how completely a polyelectrolyte chain is embraced by a dendrimer is very relevant for applications related to drug and gene delivery. To characterize this property, we chose to calculate the fraction of chain beads that are condensed on a dendrimer 


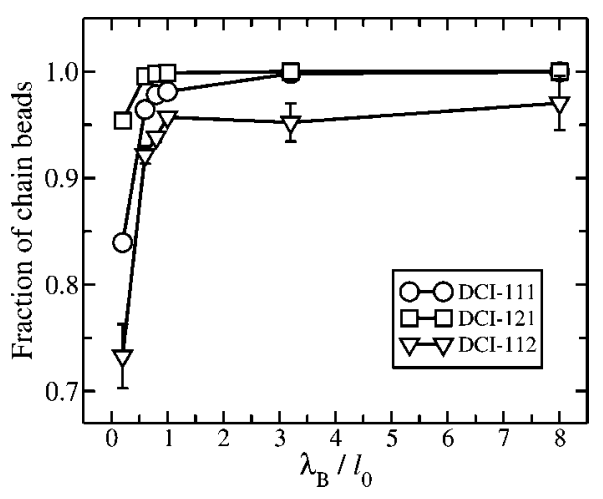

Figure 8. Average fraction of chain beads condensed on a charged dendrimer as a function of the Bjerrum length $\lambda_{\mathrm{B}}$.

(see Figure 8). As one can expect, a complex turns out to be rather loose when electrostatic interactions are weak, $\lambda_{\mathrm{B}}=0.2 l_{0}$. This is manifested as rather substantial chain tails not adsorbed on a dendrimer. These tails disappear very quickly as the Bjerrum length is increased. Already for $\lambda_{\mathrm{B}} \sim l_{0}$ the polyelectrolyte chains in systems with monovalent counterions (DCI111 and DCI-121) are almost completely condensed on a dendrimer. Remarkably, in the case of divalent ions (DCI-112), there is a small fraction of chain beads that never condense (Figure 8). We attribute this effect to strong attractive interactions between a polyelectrolyte chain taken up by a dendrimer and the chain's divalent counterions. Indeed, at larger values of $\lambda_{\mathrm{B}}$ at least one divalent counterion is always found to adsorb on a chain in complex (see Figure 5, top).

To further analyze the position of a guest chain in a complex, Figure 9 depicts component-wise distributions for the number of beads in complexes as a function of the radial distance $r$ from the center of mass (CM) of the dendrimer; here we focus on the most interesting case $\lambda_{\mathrm{B}}=l_{0}$ as it can directly be related to aqueous solution conditions. First, one finds that a divalent chain (DCI-121) is best localized within the dendrimer interior as it is characterized by the narrowest bead distribution. The widest distribution is observed for a monovalent chain with divalent counterions (DCI-112) which is a natural consequence of the above-mentioned strong electrostatic attraction between the chain and its counterions. Similarly to previous studies, ${ }^{23}$ the dendrimer beads of the terminal G4 shell are found to be broadly distributed, implying considerable back-folding. Dendrimer counterions are condensed on a dendrimer to a large degree, while chain counterions are mainly located outside the dendrimer.

It is noteworthy that for the dendrimer-chain systems with monovalent ions the maxima of bead distributions for a chain and for dendrimer counterions are localized on the opposite sides with respect to the peak of charged terminal dendrimer beads, the chain beads being located closer to the dendrimer center (see Figure 9). This strongly suggests that a polyelectrolyte chain can be accommodated in the dendrimer interior much easier compared to counterions. This is most likely because the entropy cost for compaction of charges linked to a chain is lower than that for a cloud of free charges. In the case of divalent ions this picture gets smeared. Ionic charges become partly "linked" as a dendrimer ion carries now a double charge, so that it can be easier accommodated inside the dendrimer. Furthermore, strong electrostatic interactions between a polyelectrolyte chain and its divalent counterions pull the chain closer to the dendrimer surface (see also Figure 8).

The simplified nature of the coarse-grained model employed here implies that care has to be taken when comparing our findings with experimental data and also with the results of atomistic simulations. In particular, the only atomic-scale MD
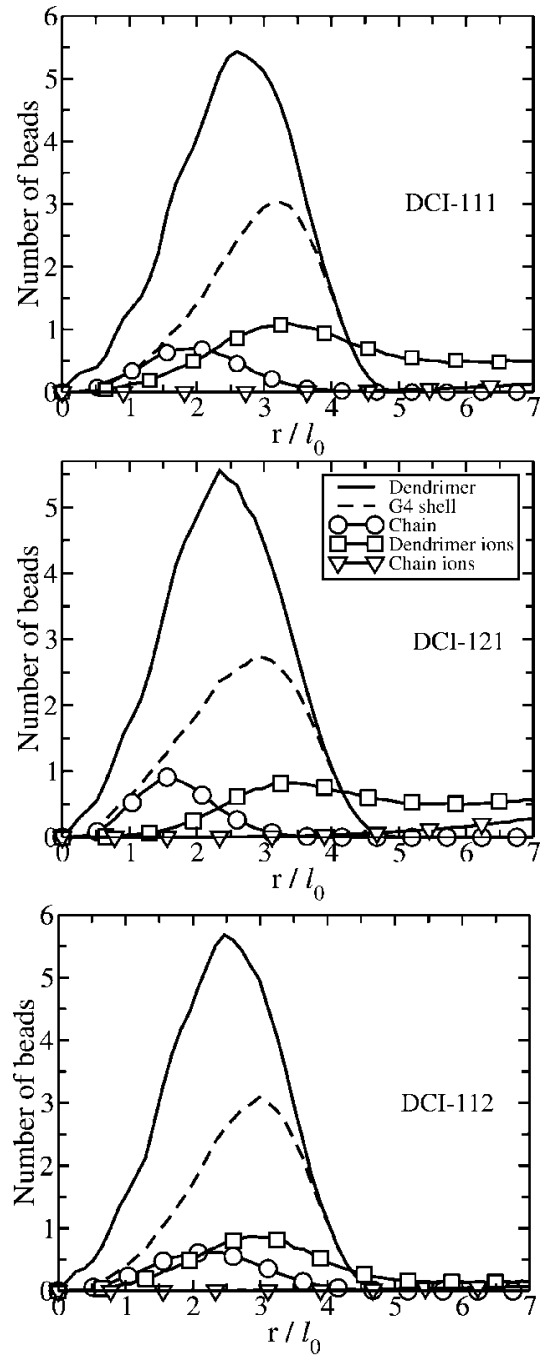

Figure 9. Distribution for the number of beads as a function of the radial distance $r$ from the center of mass of the dendrimer. Shown are results for a dendrimer, a chain, charged beads of the terminal G4 shell, and counterions of the dendrimer and the chain.

study of dendrimer-DNA complexes ${ }^{31}$ show that a ssDNA fragment in a complex is located considerably far away from the dendrimer center as compared to the picture revealed in our simulations (see Figure 9). There might be several reasons for such a discrepancy. First, our model of a dendrimer as well as a polyelectrolyte chain is a flexible one; improvement of the model through introducing angle and torsional potentials should lead to additional steric constraints which would hinder tight complexation. Note, however, that these changes should be done without major modifications to the persistence length, since the model employed in this work is consistent with the persistence length found in experiments. Second, a chain fragment in our case is much shorter than that considered in ref 31 ; this factor also can facilitate dendrimer-chain complexation. In general, the sensitivity of the results presented in the paper to the rigidity of a polyelectrolyte chain is a very relevant problem to be addressed in the future: employing a chain with large persistence length could shed light on complexation of PAMAM dendrimer(s) with double-stranded DNA.

\section{Concluding Remarks}

We have performed a systematic molecular dynamics study of complexation between cationic dendrimers of the fourth generation and oppositely charged linear polyelectrolytes. We employed a generic, coarse-grained model for the dendrimer 
and the chain; the counterions and solvent molecules were explicitly incorporated into the model. The focus in this work has been on relatively short polyelectrolyte chains, whose charged nature included both mono- and divalent cases. Additionally, for reason for completeness, we also varied ion valency by considering both mono- and divalent counterions. Our main objective has been to shed light on how the strength of electrostatic interactions affects the properties of dendrimer-chain complexes often used as a means in drug and gene delivery. To the best of our knowledge, this is the first coarse-grained computational study for complexes between dendritic and linear polyelectrolytes, correctly accounting for all principal charges in the system including counterions.

We found that dendrimer-chain complexation leads to a remarkable compaction of both charged dendrimers and polyelectrolyte chains. While the size of a chain in a complex is only slightly sensitive to the Bjerrum length, a dendrimer (and therefore a complex as a whole) demonstrates pronounced shrinking in size as the strength of electrostatic interactions is increased. This is due to condensation of dendrimer counterions. A dendrimer-chain complex efficiently screens a polyelectrolyte chain from its counterions; relevant interactions of a chain are observed only with its divalent counterions. Furthermore, complex formation leads to noticeable $(\sim 40 \%)$ dehydration of a polymer chain, the dehydration being enhanced by an increase in the strength of electrostatic interactions.

To summarize, our findings suggest that charged dendrimers have several useful characteristics. They are able to provide guest polyelectrolyte chains with a host, the resulting complex being a compact one. Further, the charged dendrimer and the associated counterions provide the polyelectrolyte chain with effective protection from the influence of surrounding medium. These properties are very important as they are well-known prerequisites for efficient vehicle-mediated drug and gene delivery. Furthermore, our study highlights the role of counterions in the structural properties of dendrimer-chain complexes; their effect continuously increases with the strength of electrostatic interactions in the system, making implicit treatment of counterions inapplicable. Further research in the area is needed to understand how the size of polyelectrolytes (the generation number of dendrimers and the length of linear polyelectrolytes) and their stiffness (the persistence length of polyelectrolyte chains) affect the structure and properties of the complexes. As physiological conditions always imply salt ions in aqueous solution, the effect of salt on "dendrimer-chain" complexes is also of considerable interest.

Acknowledgment. We thank Mikko Karttunen for discussions at the early stage of this work. This work has been supported by the Russian Foundation of Basic Research (Projects 08-03-00150 and 08-03-00565). We also acknowledge the support by INTAS (Project YS 05-109-4111 (S.V.L.)), fourth Program of the Division of Chemistry and Material Science of the Russian Academy of Sciences (S.V.L.), and the Academy of Finland (I.V.). The simulations were performed at the Finnish IT Center for Science and on the HorseShoe (DCSC) supercluster at the University of Southern Denmark.

\section{References and Notes}

(1) Svenson, S.; Tomalia, D. A. Adv. Drug Delivery Rev. 2005, 57, 21062129.

(2) Fréchet, J. M. J. Science 1994, 263, 1710-1715.

(3) van Duijvenbode, R. C.; Borkovec, M.; Koper, G. J. M. Polymer 1998, $39,2657-2664$

(4) KukowskaLatallo, J. F.; Bielinska, A. U.; Johnson, J.; Spindler, R.; Tomalia, D. A.; Baker, J. R. Proc. Natl. Acad. Sci. U.S.A. 1996, 93, 4897-4902.

(5) Esfand, R.; Tomalia, D. A. Drug Discovery Today 2001, 6, 427-436.
(6) Cloninger, M. J. Curr. Opin. Chem. Biol. 2002, 6, 742-748.

(7) Dufes, C.; Uchegbu, I. F.; Schatzlein, A. G. Adv. Drug Delivery Rev. 2005, 57, 2177-2202.

(8) Malik, N.; Evagorou, E. G.; Duncan, R. Anti-Cancer Drugs 1999, $10,767-776$.

(9) Nishikawa, M.; Huang, L. Hum. Gene Ther. 2001, 12, 861-870.

(10) Roberts, J. C.; Bhalgat, M. K.; Zera, R. T. J. Biomed. Mater. Res. 1996, 30, 53-65.

(11) Zinselmeyer, B. H.; Mackay, S. P.; Schatzlein, A. G.; Uchegbu, I. F. Pharm. Res. 2002, 19, 960-967.

(12) Boas, U.; Heegaard, P. M. H. Chem. Soc. Rev. 2004, 33, 43-63.

(13) Welch, P.; Muthukumar, M. Macromolecules 1998, 31, 5892-5897.

(14) Lee, I.; Athey, B. D.; Wetzel, A. W.; Meixner, W.; Baker, J. R. Macromolecules 2002, 35, 4510-4520.

(15) Lyulin, S. V.; Evers, L. J.; van der Schoot, P.; Darinskii, A. A.; Lyulin, A. V.; Michels, M. A. J. Macromolecules 2004, 37, 3049-3063.

(16) Lyulin, S. V.; Darinskii, A. A.; Lyulin, A. V.; Michels, M. A. J. Macromolecules 2004, 37, 4676-4685.

(17) Terao, T.; Nakayama, T. Macromolecules 2004, 37, 4686-4694.

(18) Maiti, P. K.; Cagin, T.; Lin, S. T.; Goddard, W. A., III Macromolecules 2005, 38, 979-991.

(19) Lin, S.-T.; Maiti, P. K.; Goddard, W. A., III J. Phys. Chem. B 2005 , 109, 8663-8672.

(20) Lee, H.; Baker, J. R.; Larson, R. G. J. Phys. Chem. B 2006, 110, 4014-4019.

(21) Maiti, P. K.; Goddard, W. A., III J. Phys. Chem. B 2006, 110, 2562825632.

(22) Galperin, D. E.; Ivanov, V. A.; Mazo, M. A.; Khokhlov, A. R. Polym. Sci., Ser. A 2005, 47, 61-65. (translated from Vysokomol. Soedin., Ser. A)

(23) Gurtovenko, A. A.; Lyulin, S. V.; Karttunen, M.; Vattulainen, I. J. Chem. Phys. 2006, 124, 094904.

(24) Lee, H.; Larson, R. G. J. Phys. Chem. B 2006, 110, 18204-18211.

(25) Giupponi, G.; Buzza, D. M. A.; Adolf, D. B. Macromolecules 2007, $40,5959-5965$.

(26) Lin, Y.; Liao, Q.; Jin, X. J. Phys. Chem. B 2007, 111, 5819-5828.

(27) Majtyka, M.; Klos, J. Phys. Chem. Chem. Phys. 2007, 9, 2284-2292.

(28) Blaak, R.; Lehmann, S.; Likos, C. N., Macromolecules 2008, in press. DOI: $10.1021 / \mathrm{ma} 800283 \mathrm{z}$.

(29) Welch, P.; Muthukumar, M. Macromolecules 2000, 33, 6159-6167.

(30) Lyulin, S. V.; Darinskii, A. A.; Lyulin, A. V. Macromolecules 2005 , 38, 3990-3998.

(31) Maiti, P. K.; Bagchi, B. Nano Lett. 2006, 6, 2478-2485.

(32) Lyulin, S.; Darinskii, A.; Lyulin, A. e-Polym. 2007, 097.

(33) Lyulin, S.; Karatasos, K.; Darinskii, A.; Larin, S.; Lyulin, A. Soft Matter 2008, 4, 453-457.

(34) Karatasos, K.; Adolf, D. B.; Davies, G. R. J. Chem. Phys. 2001, 115, $5310-5318$.

(35) Stevens, M. J.; Kremer, K. J. Chem. Phys. 1995, 103, 1669-1690.

(36) Darden, T.; York, D.; Pedersen, L. J. Chem. Phys. 1993, 98, 1008910092.

(37) Essman, U.; Perera, L.; Berkowitz, M. L.; Darden, T.; Lee, H.; Pedersen, L. G. J. Chem. Phys. 1995, 103, 8577-8592.

(38) Dünweg, B.; Kremer, K. J. Chem. Phys. 1993, 99, 6983-6997.

(39) Chang, R.; Yethiraj, A. J. Chem. Phys. 2003, 118, 6634-6647.

(40) Berendsen, H. J. C.; Postma, J. P. M.; van Gunsteren, W. F.; DiNola, A.; Haak, J. R. J. Chem. Phys. 1984, 81, 3684-3690.

(41) Ryckaert, J. P.; Ciccotti, G.; Berendsen, H. J. C. J. Comput. Phys. 1977, 23, 327-341.

(42) Lindahl, E.; Hess, B.; van der Spoel, D. J. Mol. Model. 2001, 7, 306317.

(43) Doi, M.; Edwards, S. F. The Theory of Polymer Dynamics; Oxford University Press: Oxford, 1986.

(44) Smith, S. B.; Cui, Y. J.; Bustamante, C. Science 1996, 271, 795-799.

(45) Murphy, M. C.; Rasnik, I.; Cheng, W.; Lohman, T. M.; Ha, T. J. Biophys. J. 2004, 86, 2530-2537.

(46) Baumann, C. G.; Smith, S. B.; Bloomfield, V. A.; Bustamante, C. Proc. Natl. Acad. Sci. U.S.A. 1997, 94, 6185-6190.

(47) Liu, S.; Muthukumar, M. J. Chem. Phys. 2002, 116, 9975-9982.

(48) Böckmann, R. A.; Hac, A.; Heimburg, T.; Grubmüller, H. Biophys. J. 2003, 85, 1647-1655.

(49) Gurtovenko, A. A.; Patra, M.; Karttunen, M.; Vattulainen, I. Biophys. J. 2004, 86, 3461-3472.

(50) Gurtovenko, A. A. J. Chem. Phys. 2005, 122, 244902.

(51) Gurtovenko, A. A.; Vattulainen, I. J. Phys. Chem. B 2008, 112, 19531962.

(52) Laguecir, A.; Stoll, S.; Kirton, G.; Dubin, P. L. J. Phys. Chem. B 2003, 107, 8056-8065. 